\title{
Comparative Analysis of Electric Field and Potential Distributions over Porcelain and Glass Insulators Using Finite Element Method
}

\author{
Adnan S. Krzma*, Mahmoud Y. Khamaira, Maruwan Abdulsamad \\ Department of Electrical and Computer Engineering, Faculty of Engineering, \\ Elmergib University, Libya
}

DOI: https://doi.org/10.21467/proceedings.2.22

* Corresponding author email: Salamaaek@Elmergib.edu.ly

\begin{abstract}
This paper investigates the electrical performance of outdoor porcelain and glass insulators when subjected to an $11 \mathrm{kV}$ AC voltage. The insulators are modeled in two dimensions, and the analysis of COMSOL software is used for the finite element simulations. The electric field and voltage distribution in the vicinity of the insulator surfaces were computed and examined. Comparative studies of field and equipotential lines for the insulators are presented under both dry clean and wet polluted conditions. The pollution layer over the insulator surfaces are assumed to be uniform with $0.5 \mathrm{~mm}$ thickness. The simulation results show that the field distribution of porcelain and glass insulators were obviously depended on the contamination conditions. The critical of high field regions on both surfaces were identified and the effect of the pollution on the electric field distribution was revealed. The results also confirmed that porcelain insulator has slightly higher field distribution near the high voltage end compared with the glass surface.
\end{abstract}

Keywords: Electric field distribution, potential distribution, finite element method, porcelain insulator, glass insulator.

\section{Introduction}

Outdoor high voltage insulators play an important role in the reliability of power system networks $[1,2]$. Ceramic insulators have been extensively used in power transmission lines and distribution systems. In fact, insulator has crucial problems when exposed to environmental and electrical conditions. The problem of the pollution is one of the phenomena affect the insulator experienced by many utilities [3,4]. The presence of the pollution layer on the insulating surface is quite common especially in costal and industrial areas. It is known that the performance of the dry insulator surface remains usually unchanged. But, when the polluted surface becomes wet, water films are formed on the insulating surface and noticeable leakage current flows [5]. The flow of leakage current on the insulator surface may increase

(C) 2018 Copyright held by the author(s). Published by AIJR Publisher in Proceedings of First Conference for Engineering

Sciences and Technology (CEST-2018), September 25-27, 2018, vol. 1.
This is an open access article under Creative Commons Attribution-NonCommercial 4.0 International (CC BY-NC 4.0) license, which permits any non-commercial use, distribution, adaptation, and reproduction in any medium, as long as the original work is properly cited. ISBN: $978-81-936820-5-0$ 
the probability of dry band arcing and this can eventually cause a complete flashover or degrade the insulator [6]. Therefore, field and potential distribution around the insulator are essential not only for the dry clean surface, but also for wet polluted condition. Moreover, knowing the electric field distribution along the insulator surface is very important for the detection of defects in the insulator. On this basis, computation of potential changes and field distribution is believed to provide valuable information on the state of the insulator and this may improve the reliability of the system [7].

In this paper, the performance of cap and pin ceramic insulators is investigated. The insulators are modelled based on the Finite Element Method (FEM) and a two dimension (2D) model COMSOL Multiphysics software is used for the simulation. The main purpose of the simulation was to evaluate the electrical performance of porcelain and glass surfaces under both dry clean and wet polluted conditions.

\section{Insulator Profile and Computational Modeling}

\subsection{Insulator Profile}

The insulator investigated in this study is a standard cap and pin suspension insulator rated at $11 \mathrm{kV}$. The insulating material and geometry of the insulator are shown in Figure 1.

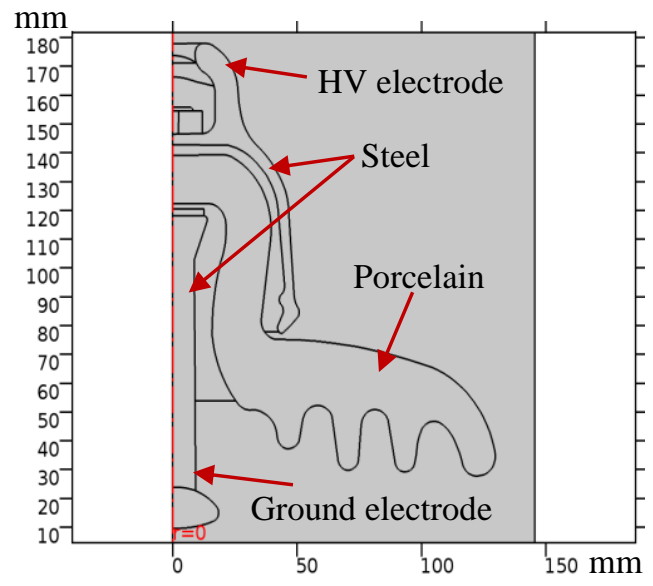

(a)

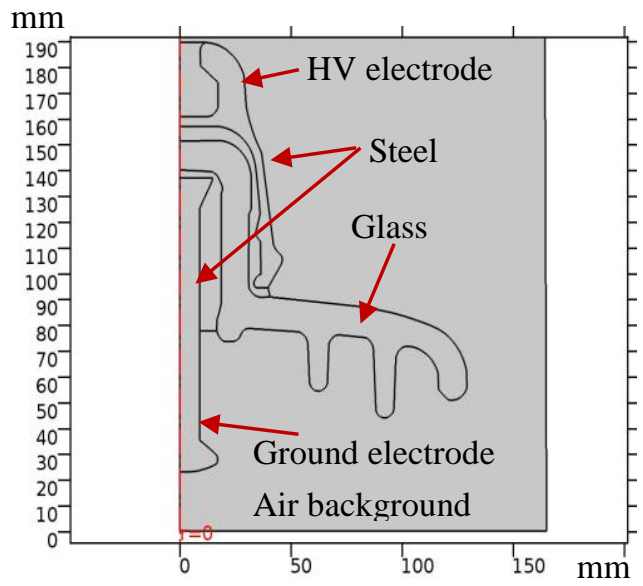

(b)

Figure 1: Cross-sectional profile of an $11 \mathrm{kV}$ cap and pin insulator: (a) porcelain (b) glass

The technical dimensions of 81022 porcelain and U100BL glass profiles are summarized in Table 1, in which $\mathrm{D}$ is the diameter of the insulating part, $\mathrm{H}$ is the configuration height and $\mathrm{L}$ is the nominal leakage distance. 
Table 1: Dimensions of 81022 porcelain and U100BL glass insulators

\begin{tabular}{|c|c|ccc|}
\hline \multicolumn{2}{|c|}{ Profile } & Material & \multicolumn{3}{c|}{ Parameters (mm) } & L \\
\hline & & & & \\
\hline
\end{tabular}

\subsection{Material Properties}

The insulators are generally comprised of two main components. The dielectric component which are porcelain and glass and the terminations which are cap and pin. The insulating housing is made of porcelain and glass materials with a relative permittivity of $\boldsymbol{\varepsilon}_{r}=4$.2. The end fitting elements used for the high voltage and ground electrodes are made of a steel. These fittings are crimped to the dielectric to support the mechanical strength of the insulator. In this simulation, the dielectric materials of porcelain and glass were assumed to be perfect insulators with a conductivity of $1 * 10^{-14} \mathrm{~S} / \mathrm{m}$. The air background region surrounding the insulators was specified with appropriate conductivity of $1 * 10^{-15} \mathrm{~S} / \mathrm{m}$. The pollution layer along the insulator surfaces is assumed to be uniform with $1.5 \mathrm{~mm}$ thickness. The conductivity of the pollution layer was adopted from the laboratory with a value of $600 * 10^{-16} \mathrm{~S} / \mathrm{m}$ and the permittivity of the layer is set to be 81 [8]. The electrical properties used for the insulator modeling are specified in Table 2 .

Table 2: Dimensions of 81022 porcelain and U100BL glass insulators

\begin{tabular}{|c|c|c|}
\hline Material & Relative permittivity, $\boldsymbol{\varepsilon}_{r}$ & Conductivity, $\sigma(\mathrm{S} / \mathrm{m})$ \\
\hline Air & 1.0 & $1 * 10^{-15}$ \\
\hline Porcelain & 4.2 & $1 * 10^{-14}$ \\
\hline Glass & 4.2 & $1 * 10^{-14}$ \\
\hline Steel & 1.0 & $1.45^{*} 10^{6}$ \\
\hline Cement & 2.09 & $1 * 10^{-14}$ \\
\hline Pollution layer & 81 & $600^{*} 10^{-6}$ \\
\hline
\end{tabular}

The high voltage (HV) terminal which represents the cap was energised with an AC voltage of $11 \mathrm{kV}$ at $50 \mathrm{~Hz}$. This r.m.s value was subjected to the insulator under both dry clean and wet polluted conditions, according to the IEC 60507 standard [9]. The lower pin terminal was connected to the ground at $0 \mathrm{~V}$. The air region surrounding the insulator is simulated effectively large to reduce its influence on the potential distribution over the insulator profile 
and near to the electrodes. The outer edges of the air region are allocated with a boundary that expected zero external current and electromagnetic sources, hence demonstrating a physical system that is in isolated open space.

\subsection{Finite Element Method (FEM)}

The procedure for creating the $2 \mathrm{D}$ symmetrical model was achieved by drawing the insulator using AutoCAD tools and saved in an extension file of $\mathrm{dfx}$ format. The insulator was then imported to the COMSOL 5.2 Multiphysics software. The simplification of using the 2D model instead of three dimensions (3D) model is preferred. This is because it saves a significant size of memory and processing time without affecting the accuracy of the simulation results. In addition, using symmetric model, only half of the insulator structure was modelled.

After completing the stage of the structure, the model is assigned with a suitable material properties and boundaries conditions as previously described. As a part of FEM system, the entire geometry of the insulator except the end fitting regions were divided into small triangular elements as shown in Figure 2. This process is called the meshing. This meshing discretisation can enhance the accuracy of the simulation results by increasing the number of meshing elements over the insulator surface where the field intensity is found to be higher.

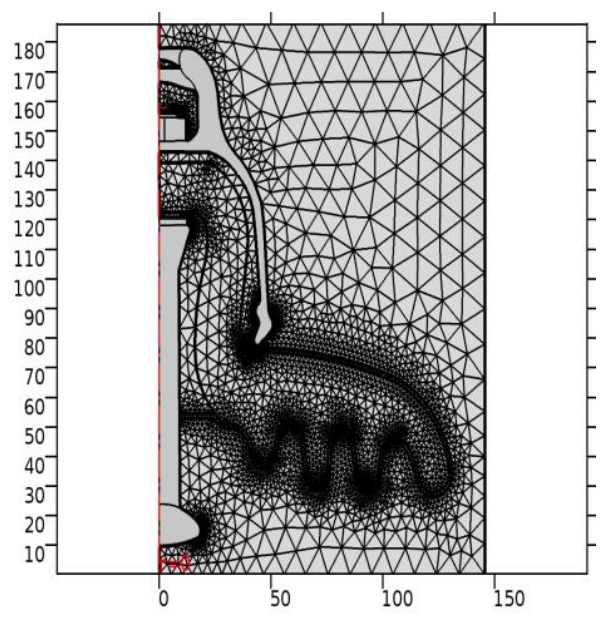

(a) Porcelain

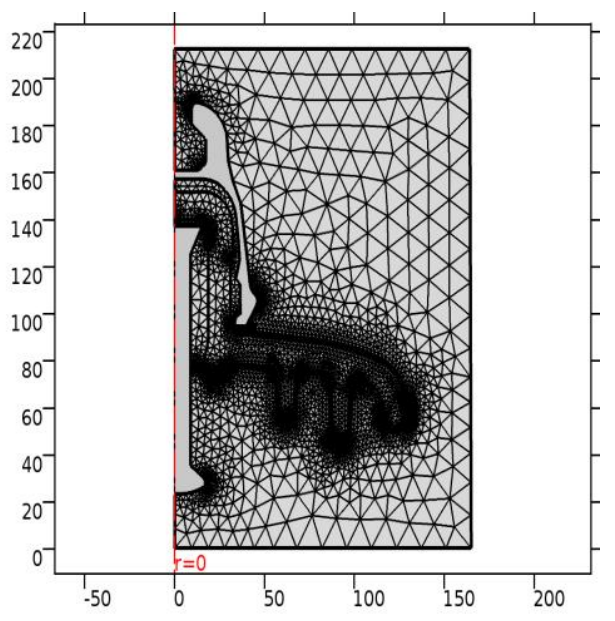

(b) Glass

Figure 2: Meshing discretisation of insulator domain (a) Porcelain (b) Glass

The insulator model is analysed using the FEM based on Quasi-Static Electric mode, which assumes that the current changing slowly with the electromagnetic field [10]. This assumption is effective for insulator and many high voltage applications that operate at $50 \mathrm{~Hz}$ power frequency. The potential and field distribution are computed by solving the differential equation in the software given by equation (1). 
$-\nabla . \partial(\varepsilon 0 \varepsilon r . \nabla r) / \partial t-\nabla \cdot(\sigma \nabla V-J e)=Q J$

WhereJe: External current density $\left(\mathrm{A} / \mathrm{m}^{2}\right)$

QJ: Current source $\left(\mathrm{A} / \mathrm{m}^{3}\right)$

$\sigma$ : Electric conductivity $(\mathrm{S} / \mathrm{m})$

$\varepsilon$ : Permittivity

\section{Simulation Results and Discussion}

\subsection{Potential Distribution}

The voltage distributions along the porcelain and glass insulators were computed for analysing and investigation studies. Figure 3(a) and Figure 3(b) respectively show the equipotential lines along the porcelain surface under both dry clean and wet polluted conditions. The lines are modeled at $5 \%$ voltage interval, therefore a total of 20 equipotential lines are plotted in the simulation results. The same action for voltage computation was achieved over the glass surface and Figure 3(c) and Figure 3(d) respectively observe the behaviour of the insulator under a certain conditions. As can be seen from both insulators, more than $75 \%$ of the voltage lines concentrated between the pin fitting and the first inner skirt. Over 15\% of the voltage contours is concentrated nearby the cap.

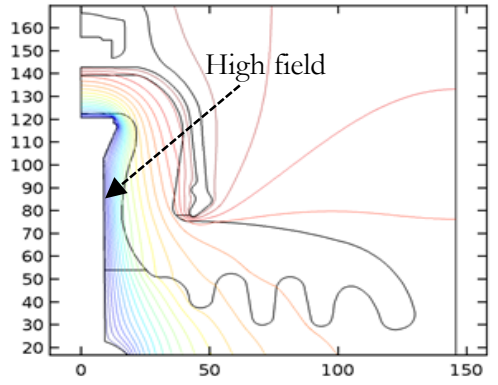

(a) Dry clean porcelain

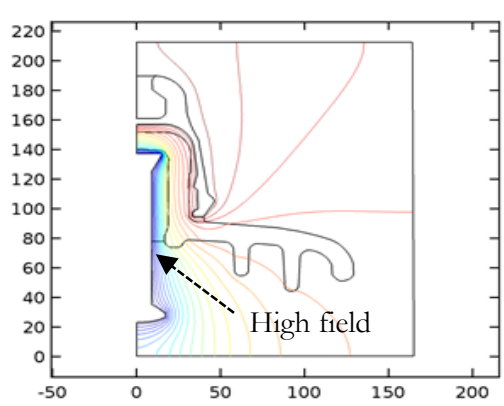

(c) Dry clean glass

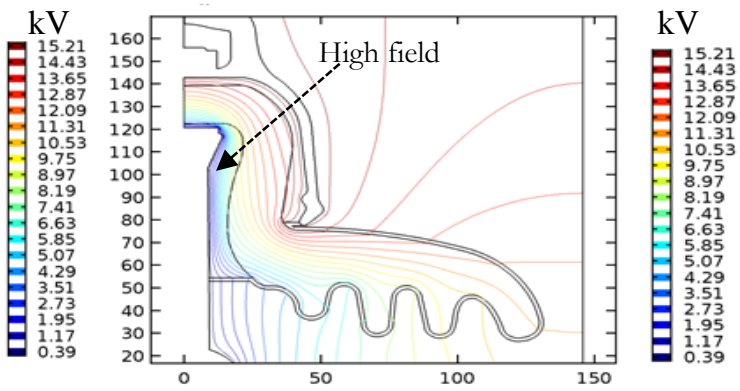

(b) Wet polluted porcelain
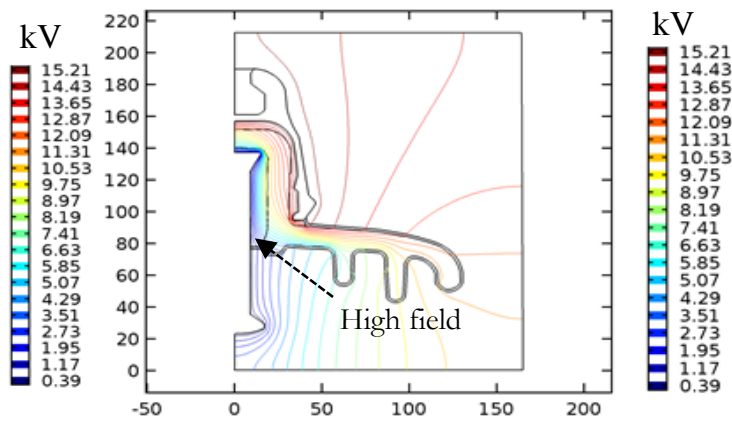

(d) Wet polluted glass

Figure 3: Equipotential contours over the surface profile of porcelain and glass insulators under dry clean and uniformly wet polluted conditions 
Krzma et al., CEST-2018, AIJR Proceedings 2, pp.176-184, 2018

The lines are commonly concentrated close to the end fitting regions and this may indicate to the highly field stress. When the insulator is polluted, the potential distributions are start to be uniform compared with those attained from the clean surface. This behaviour happens due to the presence of the pollution layer which helps to redistribute the potential lines widespread over the surface. The computed voltage profile along the porcelain and glass insulators under both surface conditions are shown in Figure 4. The leakage path of the insulator is measured along the surface, starting from the ground and termination at the HV end. An increase trend is observed for both graphs when shifting to the energisation end.

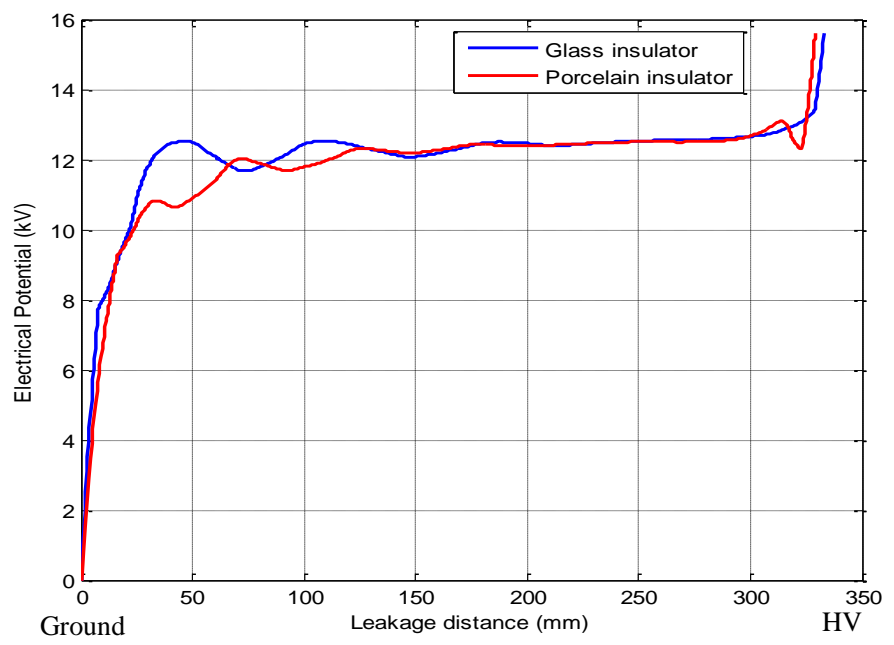

(a) Dry clean surface

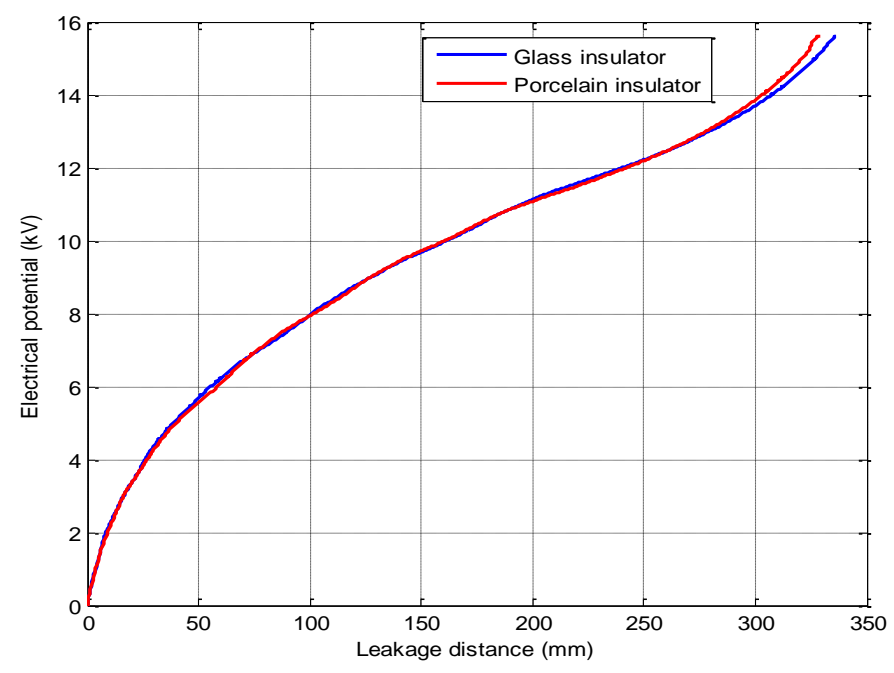

Ground

(b) Wet polluted surface

HV

Figure 4: Voltage profiles along the porcelain and glass insulators: (a) dry clean, and (b) wet polluted

Proceedings of First Conference for Engineering Sciences and Technology (CEST-2018), vol. 1 
Comparative Analysis of Electric Field and Potential Distributions over Porcelain and Glass Insulators Using Finite Element Method

For dry clean insulators, a steep rise in the potential is observed up to $11 \mathrm{kV}$ for the porcelain and $12 \mathrm{kV}$ for the glass at a surface distance of $40 \mathrm{~mm}$ nearby the ground end. The clean surface also shows curve undulations because of the individual lines cross the insulator at more than one points. These undulations are accompanied by electric field reveal over the insulator. For wet polluted conditions, the water droplets hit the top of the polluted surface and local variations in the resistive current can occur. This behaviour may redistribute the voltage profile and extremely high field can be initiated.

\subsection{Electric Field Distribution}

Figure 5 and Figure 6 show respectively the plot results of field distributions along the porcelain and glass insulators under dry clean and wet polluted surface conditions. The simulated field was represented the tangential electric field along the insulator surface. The graphs, in general, exhibit a similar tendency of field distributions along the surface profiles. Peaks can be observed in the rate of change of the tangential field at both HV and ground ends. These peaks confirmed the equipotential results concerning the high field regions. Under wet polluted condition, the tangential field attained on both surfaces was higher compared with the dry clean case. The highest field recorded was near to the metal fittings with magnitude value of $3.2 \mathrm{kV} / \mathrm{cm}$. However, away from the metal fittings along the surface distance of the insulators, the trend of the electric field was reduced to a lower value.

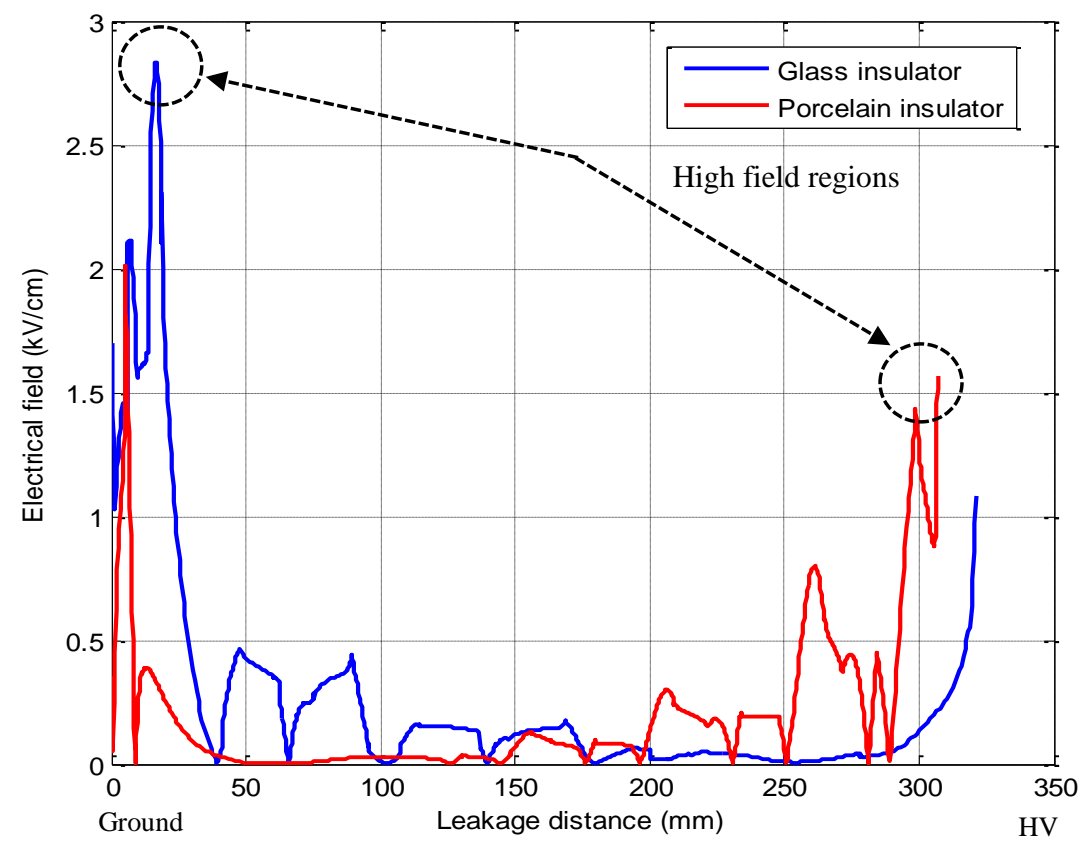

Figure 5: Field distributions over the surface of porcelain and glass insulators under dry clean condition 
Porcelain insulator exhibit a significant increase in the field near to the HV terminal compared with the glass. This can be observed under both dry clean and wet polluted conditions. Therefore, the presence of the pollution layer can increase the possibility of the electric field at the insulator surface and this may lead to the surface heating and eventually to the flashover.

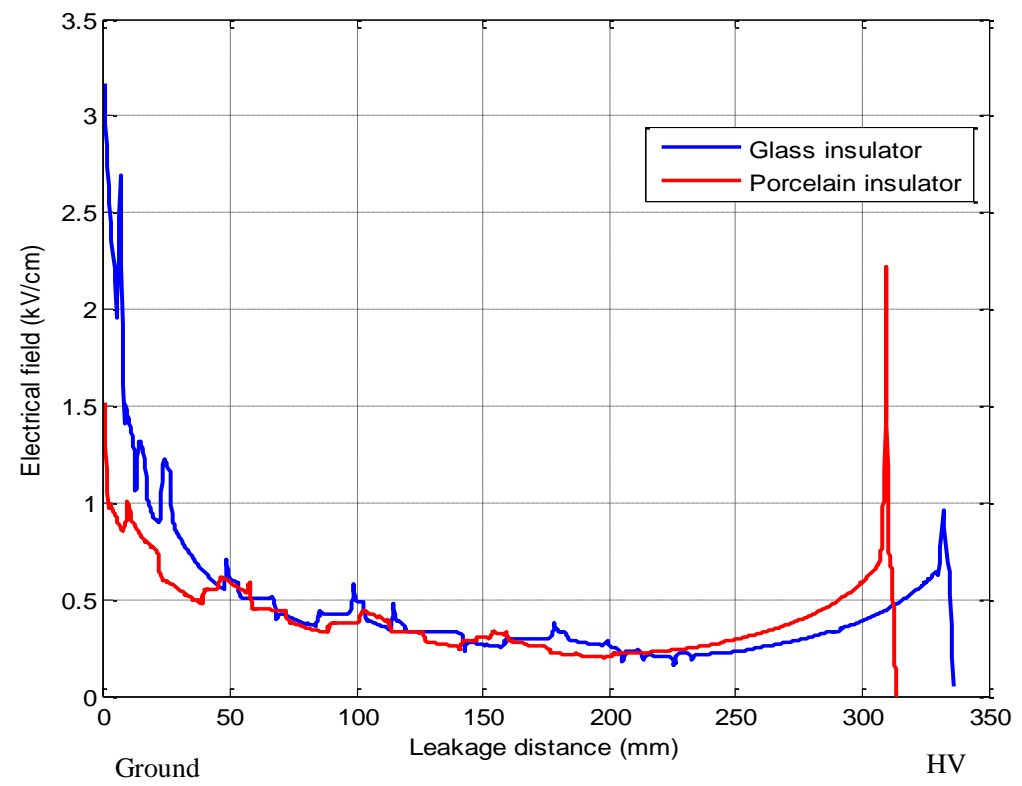

Figure 6: Field distributions over the surface of porcelain and glass insulators under wet polluted condition

\section{Conclusions}

The electrical performance of porcelain and glass insulators was investigated in this paper. The potential and field distributions over the insulator surfaces were studied using the Finite Element Method COMSOL Multiphysics Software. An ideal model has been adopted for an easily simulation the laboratory test conditions. The tangential field was performed on the insulator surfaces for both dry clean and wet polluted conditions. The result reveals that the highly field region was observed on the polluted insulators as expected. At the high voltage end region where is the extremely field stress, glass insulator offers better electrical performance compared with the porcelain. These all useful information might be worth to understand the insulator properties and to choose an appropriate design.

\section{References}

[1] J. S. T. Looms. Insulators for High Voltages. London, United Kingdom: Peter Peregrinus Ltd, 1988, pp. 2-12.

[2] R. S. Gorur, E. A. Cherney, and J. T. Burnham, Outdoor Insulators, Ravi S. Gorur, Inc., Phoenix, Arizona, USA, 1999.

[3] S. Chakravorti and H. Steinbigler, "Boundary element studies on insulator shape and electric field around HV insulators with or without pollution," IEEE Transactions on Dielectrics and Electrical Insulation, vol.7, no.2,

Proceedings of First Conference for Engineering Sciences and Technology (CEST-2018), vol. 1 
Comparative Analysis of Electric Field and Potential Distributions over Porcelain and Glass Insulators Using Finite Element Method pp.169-176, Apr 2000.

[4] A. S. Krzma, M. Albano, and A. Haddad, "Comparative performance of $11 \mathrm{kV}$ silicone rubber insulators using artificial pollution tests, "in 2015 50th International Universities Power Engineering Conference (UPEC),Stoke On Trent, United Kingdom, pp. 1-6, 2015.

[5] CIGRE Taskforce 33.04.01: "Polluted insulators: review of current knowledge," CIGRE technical brochure 158, June 2000

[6] A. S. Krzma, M. Albano, and A. Haddad, "Flashover influence of fog rate on the characteristics of polluted silicone rubber insulators," in 2017 52th International Universities Power Engineering Conference (UPEC), Crete, Greece, pp. 1-6, 2017.

[7] J. L. Rasolonjanahary, L. Krahenbuhl, and A. Nicolas, "Computation of electric fields and potential on polluted insulators using a boundary element method," IEEE Transactions on Magnetics, vol.28, no.2, pp.1473-1476, Mar 1992.

[8] M. Albano, A. S. Krzma, R. T. Waters, H. Griffiths, and A. Haddad, "Artificial pollution laver characterization on conventional and textured silicone-rubber insulators," in The 19th International Symposium on High Voltage Engineering (ISH), Pilsen, Czech Republic, 2015.

[9] IEC 60507:2013, 'Artificial pollution tests on high-voltage ceramic and glass insulators to be used on a.c. systems', 3rd edition.

[10] COMSOL Multiphysics User's Manual," ed: Version 5.2. 